\title{
Experimental complex for multi-component registration of the EAS in a wide energy range $\left(10^{15}-10^{19} \mathrm{eV}\right)$
}

\section{I.I. Yashin ${ }^{* 1}$, M.B. Amelchakov1, N.S. Barbashina ${ }^{1}$, A.G. Bogdanov',} A.A. Borisov ${ }^{1,2}$, A. Chiavassa ${ }^{1,3}$, R.M. Fakhrutdinov ${ }^{1,2}$, D.M. Gromushkin ${ }^{1}$, V.V. Kindin ${ }^{1}$, S.S. Khokhlov ${ }^{1}$, R.P. Kokoulin ${ }^{1}$, K.G. Kompaniets ${ }^{1}$, A.S. Kozhin ${ }^{1,2}$, G. Mannocchi ${ }^{4}$, A.A. Petrukhin ${ }^{1}$, O. Saavedra ${ }^{3}$, G. Trinchero ${ }^{4}$, I.A.Shulzhenko ${ }^{1}$, V.V. Shutenko', Yu.V. Stenkin ${ }^{1,5}$, E.A. Zadeba ${ }^{1}$

${ }^{1}$ National Research Nuclear University MEPhI (Moscow Engineering Physics Institute), 115409, Moscow, Russia

${ }^{2}$ RF SSC Institute of High Energy Physics, 142281, Protvino, Russia

${ }^{3}$ Dipartimento di Fisica dell' Universita di Torino et INFN, 10125, Torino, Italy

${ }^{4}$ Astrophysical Observatory of Turin, INAF, Torino, Italy

${ }^{5}$ Institute for Nuclear Research of RAS, 117312, Moscow, Russia

E-mail: iiyashin@mephi.ru

The Experimental complex (EC) NEVOD includes a number of unique experimental facilities for studies of main components of cosmic rays on the Earth's surface. The complex is used for basic research of CR flux characteristics and their interactions in the energy range $10^{15}-10^{19} \mathrm{eV}$. To extend the experimental capabilities, nowadays new large-scale detectors are being deployed around the EC NEVOD: an array for the EAS registration (NEVOD-EAS), a detector of atmospheric neutrons (URAN) and a large-area coordinate-tracking detector (TREK). The description of experimental capabilities of the complex is given, and first results of simultaneous detection of various EAS components are discussed.

The 35th International Cosmic Ray Conference - ICRC2017

12-20 July, 2017

Bexco, Busan, Korea

${ }^{*}$ Speaker 


\section{Introduction}

The Experimental complex (EC) NEVOD is located in the campus of MEPhI (Moscow) $\left(55.7^{\circ} \mathrm{N}, 37.7^{\circ} \mathrm{E}, 173 \mathrm{~m}\right.$ a.s.1.) and is intended for basic and applied research using cosmic rays $(\mathrm{CR})$ on the Earth's surface in a record-wide energy range of CR primary particles $\left(10^{9}\right.$ to $10^{19} \mathrm{eV}$ ). A distinctive feature of the complex is the possibility of detection of different components of cosmic rays at ground level including groups of quasi-parallel muons at large zenith angles, up to the horizon. During 2002 - 2007 experimental series at the EC NEVOD, a new approach to the study of primary cosmic rays based on a new EAS variable - the local muon density spectra (LMDS) - has been developed [1,2]. The measurements were conducted with main detectors of the complex - the large volume Cherenkov water detector NEVOD [3] and the large area coordinate detector DECOR. The analysis of the measured LMDS revealed a significant excess of multi-muon events generated by CR with energies $10^{15}-10^{18} \mathrm{eV}$ in comparison with calculated on the basis of modern models of hadron interactions even at the assumption of the pure iron composition of the CR spectrum [4]. This problem, named "the muon puzzle", was also noticed in other experiments $[5,6,7]$.

The energy spectrum and composition of CR flux with energies above $10^{15} \mathrm{eV}$ are the result of recalculation of the EAS parameters measured at the observation level on the basis of certain models. However, most of the EAS characteristics simultaneously depend on all these unknowns and, in addition, on a model of hadron-nuclear interactions, the parameters of which are obtained on the basis of extrapolation of accelerator data to the region of ultrahigh energies. To solve this problem, modern EAS detectors provide simultaneous measurement of different EAS components: $N_{\mathrm{e}}$ and $N_{\mu}$, energy deposit of EAS core $\Delta E_{h}$, EAS cascade curve and maximum of EAS development $X$ max. Recently, at the experimental complex NEVOD the techniques of the detection of new components of EAS have been also developed: local muon density spectra (LMDS) and $N_{\mathrm{n}}$ - number of EAS neutrons [8]. A joint analysis of these components gives more reliable estimates of the characteristics of the PCR flux. But there remains the problem of correct estimation of parameters of hadronic interactions in which EAS are produced. Important information about this could be obtained from data on the energy characteristics of the muon component.

The development of the experimental complex NEVOD is targeted to creating of new detection systems, that together with existing installations would provide multi-component detection of EAS, additionally including data on muon bundles in a wide range of zenith angles, their energy losses in the Cherenkov water calorimeter and neutron component.

\section{Present status of the experimental complex NEVOD}

The complex NEVOD [9] includes several unique detection systems for the registration of the main components of cosmic rays at ground level. The layout of the NEVOD-DECOR complex is shown in Fig. 1 (left). The basis of the complex is the Cherenkov water detector (CWD) with volume $2000 \mathrm{~m}^{2}$ of purified water $\left(9 \times 9 \times 26 \mathrm{~m}^{3}\right)$. CWD NEVOD is a multipurpose detector designed for the registration of all main components of CR on the Earth's surface. The detection system of the NEVOD is represented by a regular spatial lattice of the quasispherical measuring modules (QSM) (see Fig. 1, right). 

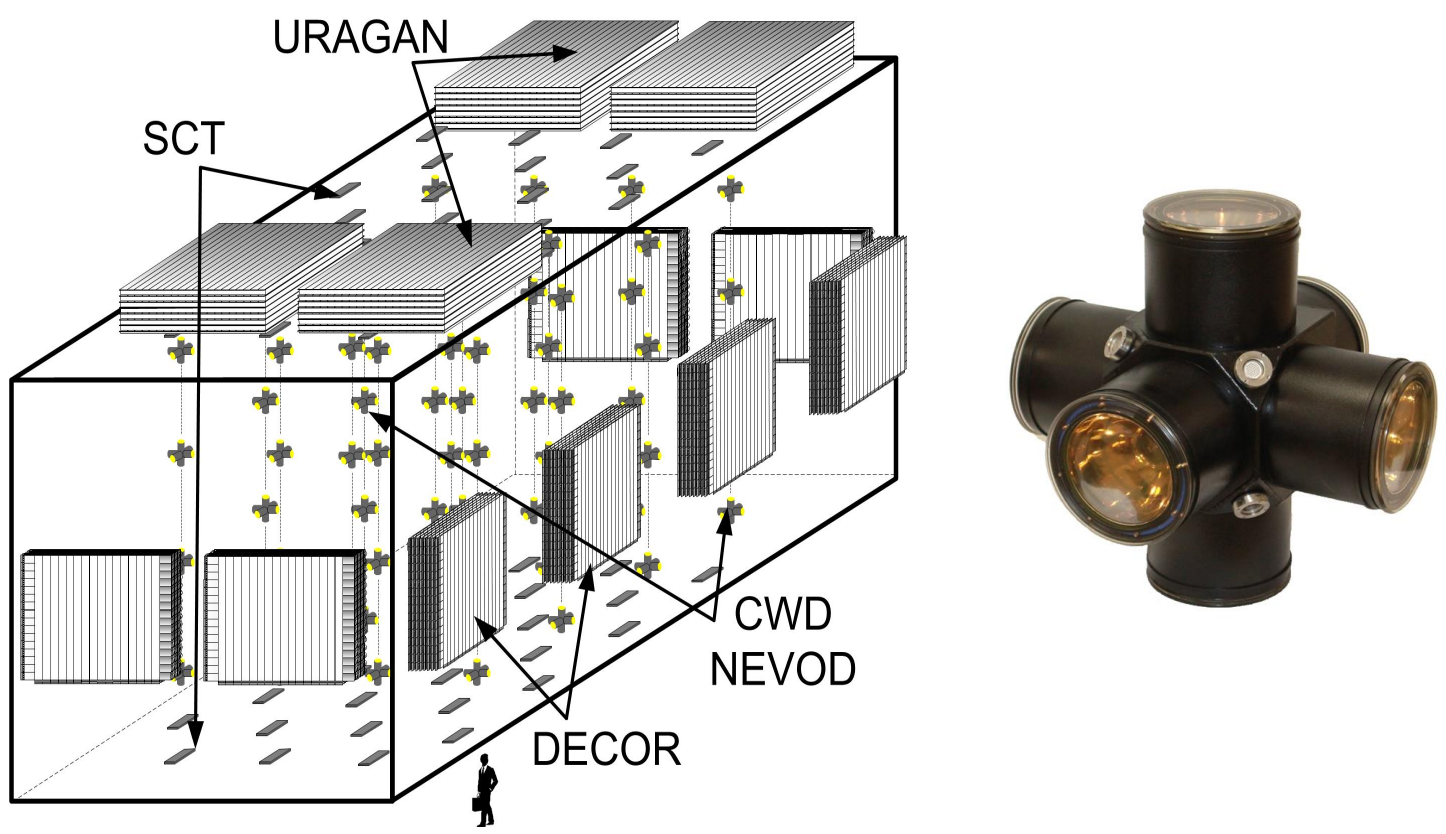

Figure 1: Experimental complex NEVOD-DECOR: left - scheme of the complex NEVOD-DECOR; right - quaisperical measuring module.

The QSM was specially designed to provide isotropic sensitivity. Each module consists of six photomultipliers FEU-200 (EKRAN company, Russia) with flat cathodes of $15 \mathrm{~cm}$ in diameter, placed in an aluminum housing and oriented along the orthogonal coordinates axes. The sum of the squares of the signals of triggered PMTs of single QSM is independent on the direction of Cherenkov light arrival. QSMs are arranged in strings with a step of $2.5 \mathrm{~m}$ along water tank and $2.0 \mathrm{~m}$ across it and over the depth. Therefore, the NEVOD measuring system has $4 \pi$ sensitivity. Having a dense lattice of QSM, the NEVOD can simultaneously perform the functions of a hodoscope, recording muon tracks in the full range of zenith and azimuth angles, and of a calorimeter detecting the energy deposit of various events in the sensitive volume. The system of scintillation calibration telescopes (SCT) is a part of EC NEVOD and allows to calibrate QSM responses and detect electromagnetic and muon components of EAS [10]. SCT consists of two parallel planes which are located on the cover and on the bottom of CWD water pool (see Fig. 1). The 40 scintillation counters with size of $40 \times 20 \times 2 \mathrm{~cm}^{3}$ are arranged in a chess order in each plane.

Precise coordinate detector DECOR is deployed around the CWD (see Fig. 1, left). DECOR represents 8 supermodules (SM, total area is $\sim 70 \mathrm{~m}^{2}$ ) vertically arranged in the galleries surrounding the water tank [11]. Each SM consists of eight vertical planes of streamer tube chambers with external strip read-out system. SM's angular and spatial resolutions are $\sim 1^{\circ}$ and $1 \mathrm{~cm}$ respectively. The main task of NEVOD-DECOR complex is the study of the muon component in wide ranges of multiplicities and zenith angles (up to the horizon).

Four SMs of the muon hodoscope URAGAN with total area about $46 \mathrm{~m}^{2}$ are horizontally deployed above the NEVOD water tank $[12,13]$. URAGAN is used for detection and analysis of angular variations of muon flux caused by various processes in the heliosphere, magnetosphere and the atmosphere of the Earth. 
To detect the lateral distribution of thermal neutrons generated by the hadron component of the EAS, around NEVOD, at the level of the upper edge of the water volume, the PRISMA-32 array is deployed [14]. PRISMA-32 detection system consists of 32 en-detectors composed of two 16-detector clusters. En-detectors are able to detect two main EAS components: electromagnetic one in a case of a synchronous passage of several charged particles, and hadronic component through thermal neutron captures. Detectors are based on a specialized inorganic scintillator, being a granulated alloy of $\mathrm{ZnS}(\mathrm{Ag})$ with $\mathrm{LiF}$, enriched up to $90 \%$ with ${ }^{6} \mathrm{Li}$ isotope. The array is triggered by the electromagnetic component of EAS, and provides information about the energy deposit (mostly electrons) and delayed neutrons accompanying the EAS within $20 \mathrm{~ms}$ after the trigger. PRISMA-32 has following characteristics: effective area of the en-detector $\sim 0.36 \mathrm{~m}^{2}$; detector spacing is $2.5 \times 5 \mathrm{~m}^{2}$; array area $\sim 500 \mathrm{~m}^{2}$, dynamic range in charged particles: 5 - 75000/det.; neutrons: 1 - 1000/det.; energy range of primary energy about $0.3-30 \mathrm{PeV}$.

\section{Tracking detector TREK}

To solve the "muon puzzle" problem, it was proposed to measure simultaneously characteristics of muon bundles and their energy deposits in the CWD which after a deep modernization represents a large volume homogeneous Cherenkov water calorimeter (CWC) [15]. However, DECOR supermodules do not overlap the whole aperture of the Cherenkov water detector and do not exclude the passage of part of muons within the gaps between SMs. Also, coordinate detector does not provide the resolution of two tracks in space better than 3 $\mathrm{cm}$. The new TREK facility [16] represents vertical planes of the multiwire drift chambers (IHEP, Protvino) [17] with $X-Y$ orientation, placed on the outside wall of the building of the complex (see Fig. 2).
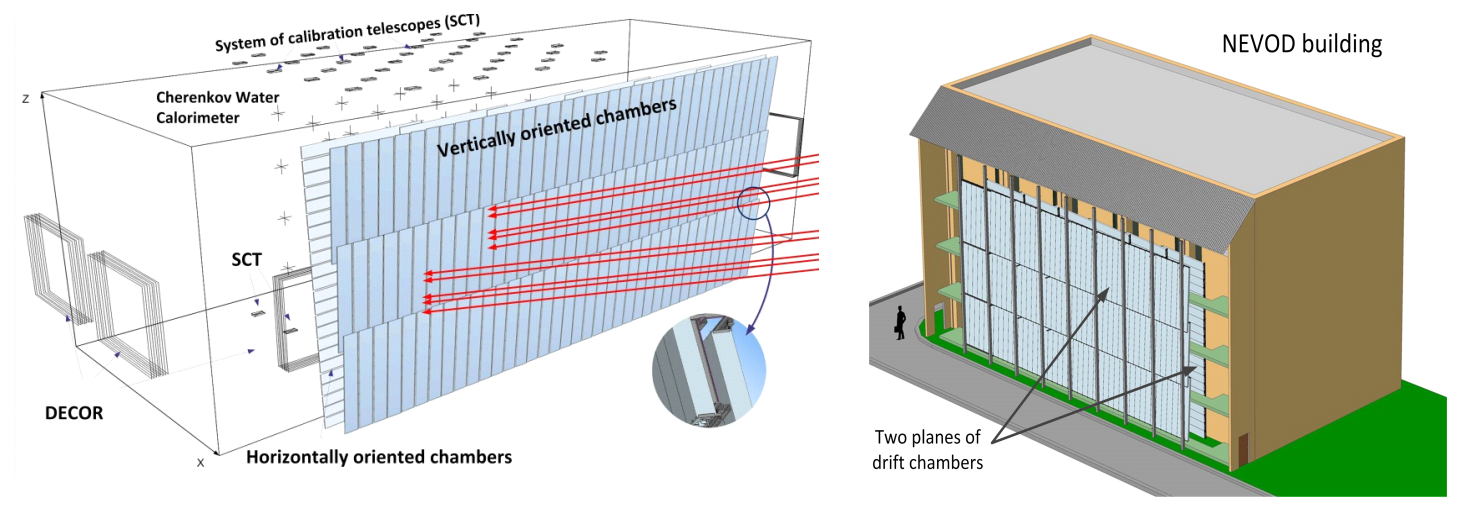

Figure 2: Left: scheme of the location of the drift chambers of the detector TREK with respect to the complex NEVOD-DECOR; right: external view of the TREK (outer wall is not shown).

Each drift chamber has a large effective area $\left(1.85 \mathrm{~m}^{2}\right)$, good spatial $(\sim 1 \mathrm{~mm})$ and angular resolution $(\sim 0.03 \mathrm{rad})$ with four measuring channels. Detector TREK will completely cover one side of the CWD NEVOD aperture and will improve the resolution of close tracks by an order of magnitude (to about $3 \mathrm{~mm}$ ). The detector will be operated as a part of the experimental complex NEVOD, in particular, together with CWD NEVOD and DECOR. Each coordinate plane will include 132 chambers. The effective area of the detector is about $270 \mathrm{~m}^{2}$. 
At present, the coordinate tracking setup on the drift chambers (CTUDC) as the prototype of full-scale TREK facility has been mounted on the opposite sides of CWD in the short galleries, one floor above the DECOR location. It consists of two coordinate planes, each containing 8 drift chambers. One of the main goals of this setup is to elaborate the conditions of the joint operation with CWD and DECOR. The Fig. 3 shows an example of a muon bundle registered simultaneously by the CTUDC and NEVOD-DECOR. The event was reconstructed separately with these setups. In the left panel of the figure, the reconstruction of the event registered by the CTUDC setup is shown. For convenience, both planes of the drift chambers located in each short gallery of the CWD are placed next to each other. Accordingly, tracks recorded with different planes are indicated by different colors. The projected zenith angle of a bundle is $65^{\circ}$ for both planes that is close to DECOR data $\left(64.6^{\circ}\right)$.

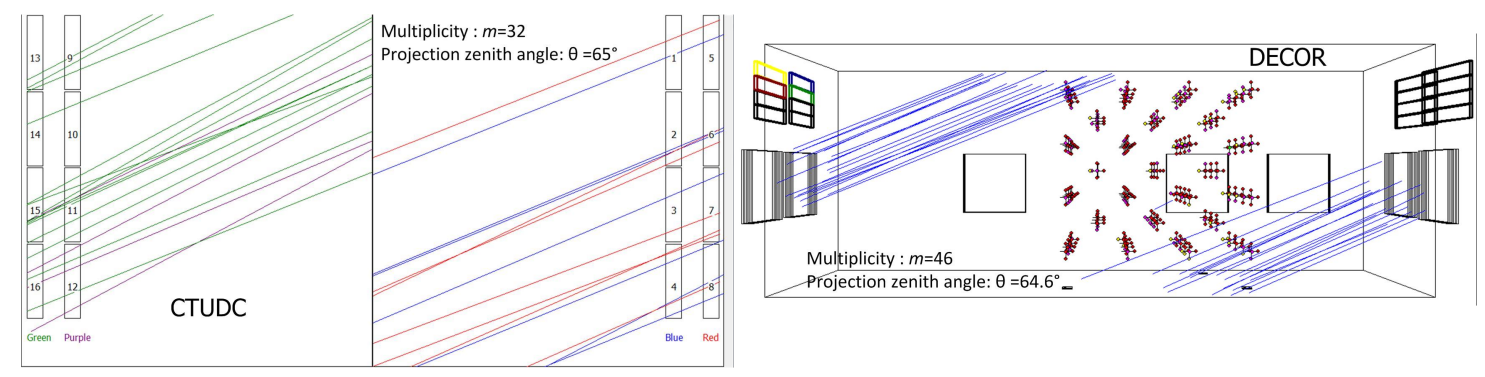

Figure 3: An example of a muon bundle registered by CTUDC (left) and DECOR (right).

\section{NEVOD-EAS array}

Another detector which began operation in the EC NEVOD is the traditional EAS detector array placed on the roofs of laboratory buildings surrounding the NEVOD and on the ground of the MEPhI campus $[18,19]$. The reason for the deployment of a traditional EAS detector is relatively low resolution for the PCR energy reconstruction via LMDS method: $\sigma_{\mathrm{lgE}} \approx 0.4$. Measuring system of the NEVOD-EAS is formed in a cluster principle. The NEVOD-EAS detector with a total area of $\sim 2 \times 10^{4} \mathrm{~m}^{2}$ will include 12 independent clusters of 16 scintillation counters in each for the detection of the EAS electron-photon component. Scintillation counters of each cluster are integrated in four detector stations (DS) and combined by a common Local Post (LP) of the detector array DAQ. Scintillation counters earlier were operated in the KASCADE-Grande detector. Scheme of the scintillation detector and the photo of the NEVODEAS detector station are shown in figures 4 and 5. Three of four detector station's counters (standard) have similar characteristics; they are used to measure the density of EAS particles, and for time measurements. The fourth counter of the detector station is characterized by a higher dynamic range (up to 10000 particles per square meter), since it contains an additional photodetector with a gain lower than that of a standard counter. NEVOD-EAS setup will provide determination of the size, position of the axis and the arrival direction of EAS with energies $10^{15}-10^{17} \mathrm{eV}$ and will give the opportunity to test a method of reconstruction of the characteristics of PCR on the basis of muon bundle registration technique by means of the DECOR detector. 


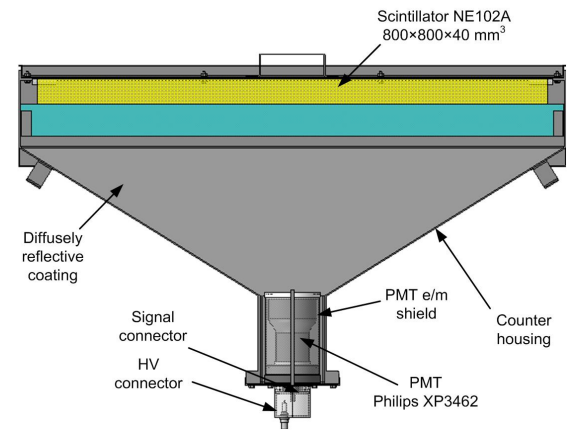

Figure 4: The scheme of the NEVOD-EAS counter.

New data obtained with NEVOD-EAS setup will allow to narrow the energy range of CR particles responsible for generation of muon bundles with certain multiplicity arriving at various zenith angles.
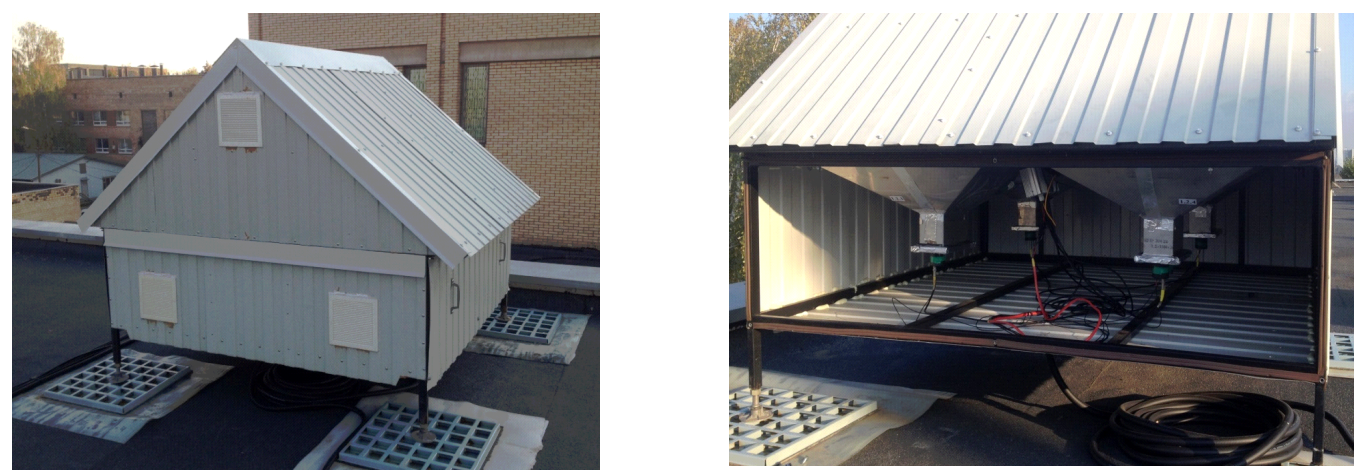

Figure 5: NEVOD-EAS detection station: left - general view of DS housing; right - wiew of detectors inside the station.

\section{URAN setup}

Currently, around NEVOD the system for registration of atmospheric neutrons - URAN - is being deployed. The first stage of the URAN array includes 72 en-detectors for simultaneous registration of electron-photon $(e)$ and neutron $(n)$ EAS components. The en-detectors are combined into independent cluster structures of 12 detectors. Clusters are located on two roofs of the laboratory buildings ( 3 clusters on each roof). The layout of the URAN setup is shown in figure 6 . The typical distance between the detectors is $4-5$ meters. The total area of the setup is $\sim 10^{3} \mathrm{~m}^{2}$. Local Post (LP) of URAN clusters provides selection of events according to the trigger conditions, digitization of amplitude information and data transmission to the Central DAQ Post via optical link. LP includes the crate with two 12-channel boards of amplitude analysis and controller, summator-multiplexer and its controller, and power supply $(+5 \mathrm{~V}$ and $\pm 12 \mathrm{~V}$ ), the mediaconverter and the LP thermostabilization system. One LP can support operation of two clusters. The Central DAQ Post of control and data acquisition ensures operation of the URAN setup in the exposition and monitoring modes, controls all LP and stores experimental data. Time synchronization of clusters with an accuracy of $10 \mathrm{~ns}$ is performed using GPS/GLONASS systems. For the registration of the EAS neutron component, a new type of detector, well-proven in the PRISMA-32 setup [14], is used. The scheme of the detector with the outer enclosure is shown in right panel of Fig. 6. 

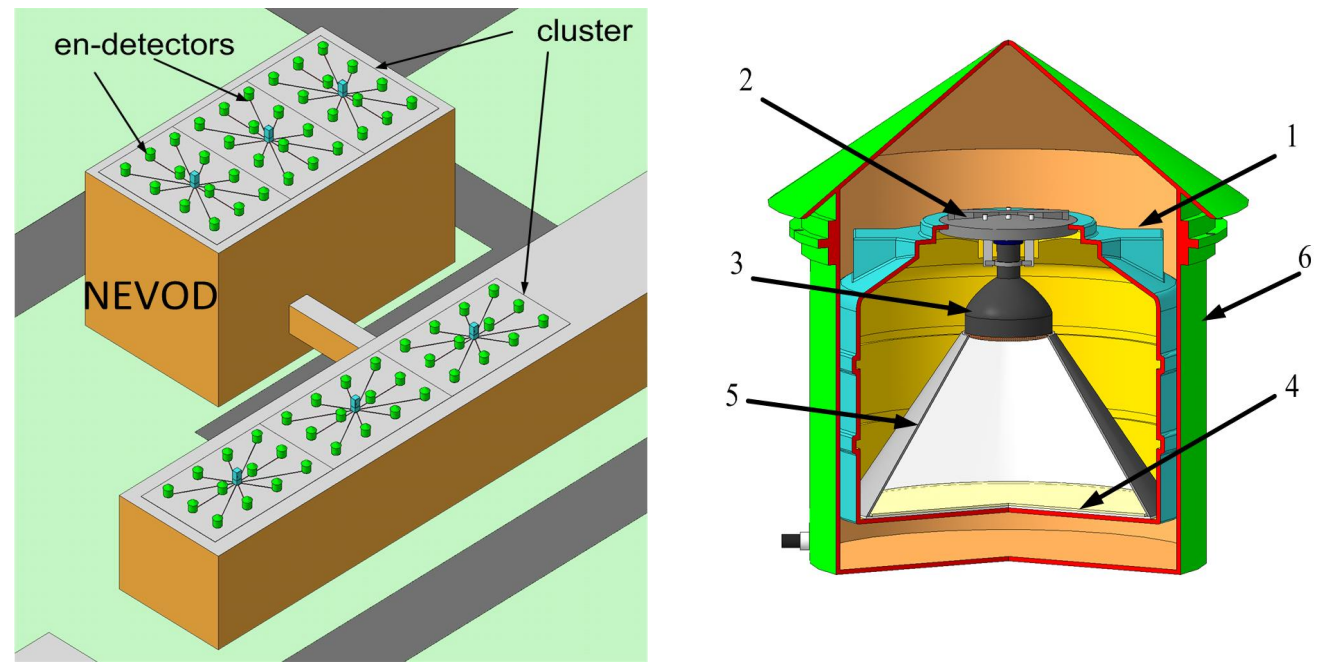

Figure 6: Scheme of the URAN setup: left - six clusters on the roofs; right - the design of en-detector; 1 light-shielding enclosure of the detector; 2 - a lid with a suspension; 3 - PMT; 4 - scintillator ZnS (Ag) + $\mathrm{B}_{2} \mathrm{O}_{3} ; 5$ - reflective cone; 6 - outer casing (galvanized steel).

The detection of thermal neutrons by scintillator $\mathrm{ZnS}(\mathrm{Ag})+\mathrm{B}_{2} \mathrm{O}_{3}$ occurs in the following capture reactions $(\sigma=3838$ barn):

$$
\begin{aligned}
&{ }^{10} \mathrm{~B}+\mathrm{n} \rightarrow{ }^{7} \mathrm{Li}+\alpha+2.792 \mathrm{MeV} \\
&{ }^{10} \mathrm{~B}+\mathrm{n} \rightarrow{ }^{7} \mathrm{Li}^{*}+\alpha+2.31 \mathrm{MeV} \\
&{ }^{7} \mathrm{Li}^{*} \rightarrow{ }^{7} \mathrm{Li}+\gamma+482 \mathrm{keV}
\end{aligned}
$$

The scintillator is made in a form of a silicon plate with the area of $0.36 \mathrm{~m}^{2}$ and thickness of $5 \mathrm{~mm}$. The thickness of the scintillator composition is about $50 \mathrm{mg} / \mathrm{cm}^{2}$ and provides thermal neutron detection efficiency of $\sim 20 \%$.

\section{Conclusion}

Equipping of the experimental complex NEVOD with new unique detectors is aimed to the developing of a new multi-component approach to the study of PCR in a very wide energy interval. Precise studies of the muon component of EAS in a wide range of zenith angles with simultaneous detection of energy deposits of muon bundles in Cherenkov water calorimeter will be conducted for the first time. Such measurements will be complemented by information on the neutron component of EAS by means of the detector also being created for these purposes for the first time.

\section{Acknowledgments}

This work has been performed at the Unique Scientific Facility "Experimental complex NEVOD" and was supported by the Ministry of Education and Science of the Russian Federation (contract RFMEFI59114X0002, and MEPhI Academic Excellence Project 02.a03.21.0005 of 27.08.2013) and by the grant of the Russian Foundation for Basic Research (project 13-02-12207-ofi-m-2013). 


\section{References}

[1] A.G. Bogdanov et al., Investigation of the properties of the flux and interaction of ultrahigh-energy cosmic rays by the method of local-muon-density spectra, Phys. Atomic Nuclei, 73 (2010) 18521869.

[2] N.S. Barbashina et al., Ultra-high energy cosmic ray investigations by means of EAS muon density measurements, Nucl. Phys. B (Proc. Suppl.), 165 (2007) 317-323.

[3] V.M. Aynutdinov et al., Neutrino water detector on the Earth's surface (NEVOD), Astrophysics and Space Science, 258 (1998) 105-115.

[4] R.P. Kokoulin et al., Local muon density spectra at large zenith angles as a probe of high-energy hadronic interaction models, Nucl. Phys. B (Proc. Suppl.), 196 (2009) 106-109.

[5] C. Grupen et al., Cosmic ray results from the CosmoALEPH experiment, Nucl. Phys. B (Proc. Suppl.), 176 (2008) 286-293.

[6] J. Abdallah et al., Study of multi-muon bundles in cosmic ray showers detected with the DELPHI detector at LEP, Astropat. Phys., 28 (2007) 273-286.

[7] G. Rodriguez et al., A measurement of the muon number in showers using inclined events detected at the Pierre Auger Observatory, EPJ Web of Conferences, 53 (2013) 07003.

[8] D.M. Gromushkin et al., Study of EAS neutron component temporal structure. Astrophys. Space Sci. Trans., 7 (2011) 115-117.

[9] V.V. Kindin et al., Cherenkov water detector NEVOD: a new stage of development, Phys. Procedia, 74 (2015) 435-441.

[10] M.B. Amelchakov et al., Measuring the spectrum of the local density of charged particles on the SCT setup, Bull. Russ. Acad. Sci.: Phys., 79 (2015) 368-370.

[11] N.S. Barbashina et al. Instrum. Experim. Techniques, 43 (2000) 743.

[12] D.V. Chernov et al., Experimental setup for muon diagnostics of the Earth's atmosphere and magnetosphere (the URAGAN project), Proc. 29th ICRC (Pune), 2 (2005) 457-460.

[13] N.S. Barbashina et al., The URAGAN wide-aperture large-area muon hodoscope, Instrum. Experim. Techn., 51 2008) 180-186.

[14] D.M. Gromushkin et al., The array for EAS neutron component detection, J.Instr., 9 (2014) 08028.

[15] V.V. Kindin et al., Cherenkov water calorimeter on the basis of quasispherical modules,, PoS(ICRC2015) (2015) 676.

[16] E.A. Zadeba et al., Status of a development of the large scale coordinate-tracking setup based on the drift chambers, J. Phys.: Conf. Ser., 632 (2015) 012031.

[17] N.I. Bozhko et al., Drift chamber for the Serpukhov neutrino detector, Nucl. Instrum. Meth. A, 243 (1986) 388-394.

[18] I.I. Yashin et al., EAS array of the NEVOD experimental complex, J. Phys.: Conf. Ser., 632 (2015) 012029.

[19] I.A. Shulzhenko et al., A proposed NEVOD-EAS installation for the detection of extensive air showers, Bull. Russ. Acad. Sci.: Phys., 77 (2013) 641-644. 